\title{
Neuronal Substrates for State-Dependent Changes in Coordination between Motoneuron Pools during Fictive Locomotion in the Lamprey Spinal Cord
}

\author{
Tim Mentel, ${ }^{1}$ Lorenzo Cangiano, ${ }^{2}$ Sten Grillner, ${ }^{2}$ and Ansgar Büschges ${ }^{1}$ \\ ${ }^{1}$ Department of Animal Physiology, Zoological Institute, University of Cologne, 50923 Cologne, North-Rhine-Westfalia, Germany, and ${ }^{2}$ Department of \\ Neuroscience, Karolinska Institutet, SE-17177 Stockholm, Sweden
}

\begin{abstract}
Locomotion relies on a precisely timed activation of sets of motoneurons. A fundamental question is how this is achieved. In the lamprey, fin and myotomal motoneurons located on the same side of the spinal cord display alternating activity during straight swimming. The neural mechanism underlying this alternation is studied here during fictive locomotion induced by superfusion with NMDA, or locomotor bursting induced by electrical stimulation. If the spinal cord is split longitudinally, each hemicord still displays rhythmic locomotor related burst activity, but now fin and myotomal motoneurons become active in-phase. The out-of-phase activation of fin motoneurons persists only when at least three segments are left intact in the rostral part of the spinal cord. Proper coordination of fin motoneurons thus requires input from contralateral rostral segments. We show that commissural excitatory interneurons with long descending axons, previously reported to be active in phase with their ipsilateral myotomal motoneurons, provide monosynaptic excitation to contralateral fin motoneurons. Together, these results strongly indicate that, although myotomal motoneurons receive their phasic excitation from ipsilateral excitatory interneurons, fin motoneurons are mainly driven from the contralateral segmental network during bilateral locomotor activity. However, during unilateral bursting, fin and myotomal motoneurons instead receive a common input, which is apparently masked during normal fictive swimming. The spinal organization thus also provides circuitry for different patterns of coordination, i.e., alternation or coactivation of the two pools of motoneurons, which may subserve different forms of locomotor behavior.
\end{abstract}

Key words: central pattern generation; fin motoneuron; intersegmental coordination; intrasegmental coordination; motor pattern generation; spinal cord

\section{Introduction}

Generation of animal locomotion is based on a well coordinated activation of different sets of motoneurons innervating muscles of the executive organs like legs or wings. In the lamprey the undulating movement of the trunk, which propels the animal through the water, is based on the activation of myotomal muscles of the body wall and the muscles moving the dorsal fins. Each of these muscles is driven by individual sets of motoneurons that display left-right alternating activity (Buchanan and Cohen, 1982; Wallén and Williams, 1984; Wallén et al., 1985; Buchanan and Grillner, 1987; Mentel et al., 2006). The dorsal fin is controlled by a thin layer of muscle fibers located on both sides of a central row of fin rays. By being active out-of-phase with the ipsilateral myotomal muscles, they counteract passive bending of

Received June 18, 2007; revised Nov. 28, 2007; accepted Nov. 28, 2007

This work was supported by Deutsche Forschungsgemeinschaft Grant Bu 857/7 and a travel grant from the Province of North Rhine-Westphalia, Germany. We are grateful to Drs. A. El Manira, R. Hill, 0. Kiehn, S. Hooper, and J. Schmidt for stimulating discussions in the course of this work and for critically reading this manuscript. We thank S. Marasigan, S. Meyen-Southard and H.-P. Bollhagen for their excellent technical assistance and S. Meyen-Southard and Z. Biro for style editing this manuscript.

Correspondence should be addressed to Dr. Ansgar Büschges, Department of Animal Physiology, Zoological Institute, University of Cologne, 50923 Cologne, Germany. E-mail: ansgar.bueschges@uni-koeln.de.

L. Cangiano's present address: Department of Psychiatry and Neurobiology, University of Pisa, Pisa 56126, Italy. DOI:10.1523/JNEUROSCI.4250-07.2008

Copyright $\odot 2008$ Society for Neuroscience $\quad$ 0270-6474/08/280868-12\$15.00/0 the fin during swimming (Rovainen and Birnberger, 1971; El Manira et al., 1996).

The basic activity pattern for swimming in the lamprey, as in other vertebrates, is generated by spinal neuronal networks referred to as central pattern generators (CPGs), which are activated by descending fibers from the brainstem (for review, see Orlovsky et al., 1999; Grillner, 2003). Ipsilateral hemisegmental glutamatergic networks of interneurons drive the rhythmic activity of myotomal motoneurons on either side of the spinal cord. Frequency is slowed down and alternating activity between contralateral myotomal motoneurons is organized by the action of glycinergic crossing interneurons (Cohen and Harris-Warrick, 1984; Cangiano and Grillner, 2003, 2005). This spinal neural network generates rhythmic, left-right alternating activity in myotomal motoneurons ( $\mathrm{mMNs}$ ), while at the same time keeping the fin motoneurons (fMNs) out-of-phase with the ipsilateral mMNs (Shupliakov et al., 1992; Mentel et al., 2006), a coordination that nonetheless displays some flexibility (Mentel et al., 2006; Porres et al., 2007). Although the generation of alternating motor output for contralateral mMNs is well understood, it is not yet known what produces and binds the fin motoneuron activity phases to the myotomal activity cycle. Morphologically, fMNs are interspersed between mMNs in the spinal cord (Shupliakov et al., 1992; Mentel et al., 2006), but they extend dendrites across the 
midline (Shupliakov et al., 1992). The out-of-phase activity is, however, not attributable to input to these crossing dendrites, because after a segmental midline lesion (three segments) out-ofphase activity of the fMNs is maintained (Mentel et al., 2006).

Here, we examine the origin of the input and timing of $\mathrm{fMNs}$ during fictive locomotion. First we show that the coordination of fMN activity in the locomotor cycle depends on the activity of crossing fibers from contralateral commissural interneurons. It is further demonstrated that the input from these commissural interneurons is excitatory and monosynaptic. Second we show that, when the contralateral network is not active, fMNs instead receive rhythmic drive from the ipsilateral hemicord. The greater flexibility in coordination enabled by this network topology may allow a richer motor repertoire and may be recruited physiologically within specific functional contexts.

\section{Materials and Methods}

Experiments were performed on adult male and female river lampreys (Lampetra fluviatilis) in the anadromous stage. Animals were kept at $4^{\circ} \mathrm{C}$ with a light/dark cycle of $12 \mathrm{~h}$ each.

Preparation and histology. The lamprey was anesthetized with MS-222 (ethyl 3-aminobenzoate methanesulfonate; $100 \mathrm{mg} / \mathrm{L}$ water; Sigma, St. Louis, MO) until pinching the tail did not initiate any reflex reactions in the animal. Then it was moved to a preparation tray (containing Sylgard gel; Dow Corning, Midland, MI) filled with oxygenated, cooled $\left(8^{\circ} \mathrm{C}\right)$ lamprey Ringer's solution (HEPES buffer) (Brodin and Grillner, 1985) and kept on ice. The animal was decapitated with a scalpel (Fine Science Tools, Foster City, CA) caudal to the gills. The tail was removed caudal to the genital pores. The ventral myotomal muscles and the inner organs were removed with scissors (Fine Science Tools). After pinning down the spinal cord/notochord, the lateral myotomal muscles were cutoff of both sides with a scalpel and the ectomeninx was removed with micro scissors (Fine Science Tools). After cutting off the ventral half of the notochord with a scalpel, a 12-14 segment piece of the caudal spinal cord/notochord preparation was pinned down in an experimental chamber and the loose fatty tissue filling the cavity between spinal cord and notochord was removed. The preparation was perfused with oxygenated Ringer's solution throughout the entire experiment. To make the cells accessible for penetration with a microelectrode, the meninx primitiva of the spinal cord was removed with fine forceps.

To study the question of whether contralateral signals affect the coordination of the mMNs and fMNs, lesion experiments were performed on spinal cord/notochord preparations. Sagittal lesions were performed as described by Cangiano and Grillner (2003). Complete longitudinal hemisections (hemicords) as well as partial longitudinal lesions (30-50\% and $50-70 \%$ ) were performed with a micro Feather ophthalmic scalpel (Feather Safety Razor, Osaka, Japan). The spinal cord was severed at 800 $\mu \mathrm{m}$ intervals along the midline with a scalpel mounted on a micromanipulator. The total percentage of the lesioned midline was evaluated after each experiment by measuring the length of the cuts under a microscope. To analyze the nature of synaptic inputs from commissural interneurons with long descending projections onto $\mathrm{AMNs}$, the pieces of spinal cord were lesioned from the caudal side up to eight segments rostrally from the recording site. Ventral root activity was recorded on both sides of the rostral intact part and up to six segments caudally on one side of the lesioned part. Fin MNs were recorded from this caudal segment intracellularly in parallel to the ventral root recordings.

Fictive swimming was induced pharmacologically by superfusion with $150 \mu \mathrm{M}$ NMDA. In other experiments, bouts of rhythmic motor activity were evoked by electrical stimulation with a single train of 10 or 20 pulses ( $2 \mathrm{~ms}$ pulse duration, $30 \mathrm{~Hz}$ frequency) delivered by a large suction electrode placed on the lateral surface of the spinal cord. To protect the tissue from damage, the electrode was lifted off after making contact so that the tip of the suction electrode was slightly above the tissue.

Staining and identification of fin motoneurons. Fin MNs were made visible for intracellular recording with fluorescence microscopy (Wild M3 with fluorescence-filter and mercury lamp; Leitz, Wetzlar, Ger- many). Fluorescein coupled dextran (FDA; 3000MW, anionic, lysine fixable; Invitrogen, Carlsbad, CA) was injected into the fin muscles $10-14 \mathrm{~d}$ before an experiment as described previously by Mentel et al. (2006). Dextran conjugates like FDA are known to be anionic and relatively inert (Haugland, 2002) and care was also taken to reduce the exposure time to the mercury lamp illumination as much as possible. Specifically, the light beam was only active for positioning the recording electrode and then was switched off throughout impalement and recording. To confirm the recording of a $\mathrm{fMN}$, a second dye was injected into the cell at the end of the experiment. The tip of the electrode was filled with 5\% tetramethylrhodamine-dextran (RDA; 3000MW, anionic, lysine fixable; Invitrogen, $5 \%$ in $0.1 \mathrm{M} \mathrm{KCl} / 3 \mathrm{M} \mathrm{KAc}$ ) and the shaft of the electrode was then filled up with $0.1 \mathrm{M} \mathrm{KCl} / 3 \mathrm{M} \mathrm{KAc}$. This dye was injected into the cell by a depolarizing current of 1-4 nA for 20-40 min. After dye injection, the spinal cord was fixed in $4 \%$ paraformaldehyde in a $0.1 \mathrm{~m}$ phosphate buffer, $\mathrm{pH} 7.4$, for $2 \mathrm{~h}$, washed $3 \times 15 \mathrm{~min}$ in $0.1 \mathrm{M}$ phosphate buffer and dehydrated in ascending ethanol series (50, 70, 90, and 100\%, $10 \mathrm{~min}$ each). The spinal cord was transferred onto a microscope slide and cleared and embedded in methyl salycilate. Optical sections of the double labeled cells were made and documented with a Zeiss (Oberkochen, Germany) 510 laser scanning confocal microscope (FDA, argon laser, excitation $488 \mathrm{~nm}$; RDA, HeNe laser, excitation $543 \mathrm{~nm}$ ).

Electrophysiology. Fin muscles are innervated by up to 20 associated motoneurons per caudal spinal segment (Mentel et al., 2006). Intracellular recordings were performed with thin-walled sharp microelectrodes (GC100TF-10; Clark Electromedical Instruments, Reading, UK) in bridge mode using an intracellular amplifier (SEC-10L; NPI, Tamm, Germany). The electrodes had a resistance of 15-32 $\mathrm{M} \Omega$ when filled with $0.1 \mathrm{~m} \mathrm{KCl} / 3$ м KAc. Retrogradely labeled fMNs were visually targeted, impaled and preliminarily identified as such by two criteria: (1) in an intact preparation superfused with NMDA, the recorded neuron's activity was out-of-phase with the ipsilateral myotomal burst activity and (2) an action potential could be recorded in an adjacent ventral root during intracellular stimulation of the penetrated neuron. In some cases, it was not possible to evoke an action potential with current injection. In these cases an antidromic spike was evoked by extracellular stimulation of the corresponding ventral root to confirm the recording of a motoneuron. The orthodromic spike traveling in the motoneuron axon and projecting through the ventral root was monitored by extracellular recording with a suction electrode made of borosilicate glass (tip diameter, $\sim 100 \mu \mathrm{m}$ ). The same electrode was used for recording mass activity in the ventral root during locomotor bursting.

In some experiments, synaptic inputs to recorded fMNs were investigated by stimulating contralateral, crossed caudally projecting interneuron axons with a stimulation electrode located six to eight segments rostrally on the midventral surface of the spinal cord and applying stimulus pulses of $0.5 \mathrm{~ms}$ duration at varying strengths according to previously established procedures (Büschges et al., 2000). Synaptic potentials recorded in motoneurons occurred at constant latency and also followed high frequency stimulation $(10 \mathrm{~Hz})$, suggesting that they were monosynaptic.

Data storage and evaluation. Sampling rates of $13.3 \mathrm{kHz}$ for extracellular recordings and $5.3 \mathrm{kHz}$ for intracellular recordings were digitized with an analog-to-digital converter CED 1401 plus (Cambridge Electronics, Cambridge, UK). The data were stored on a personal computer (Pentium-II, $350 \mathrm{MHz}$ ) and analyzed with CED Spike 2 software (version 5.12). The activity of motoneurons during NMDA induced fictive locomotion as well as in the rhythmical bouts evoked by electrical stimulation was evaluated by marking myotomal burst onset and termination of burst activity using a Spike2 script. The results were checked by visual inspection for each burst. From these markers, the exact time of each midburst was calculated using another Spike2 script. For evaluating the phasing of membrane potential oscillations of fMNs it was necessary, in some cases, to remove individual action potentials from the intracellular recording, because their large amplitude would have interfered with the analysis. A Spike2 script was used for detecting the peaks of the action potentials. Each action potential was substituted by a linear interpolation of the voltage record for its entire duration (5-10 ms before and after the peak of the action potential). The length of a locomotor cycle was defined 
by the midpoint of the extracellularly recorded ipsilateral $\mathrm{mMN}$ burst in the ventral root to the midpoint of the following burst.

Cross-correlation analysis of membrane potential oscillations versus ventral root bursting was performed as described previously by Cangiano and Grillner (2005). The ventral root trace was rectified and smoothed by a running average window $20 \mathrm{~ms}$ wide. A 15-90 s stretch of recording was then subdivided in sections of $0.5 \mathrm{~s}$ each ( $1 \mathrm{~s}$ for the correlations in Fig. 7). Cross-correlations were computed for every section and, from the entire set, an average and SE were obtained. Membrane potential oscillations were considered significantly correlated to ventral root bursting if the central peak of the average cross-correlation exceeded four times the SE (Kremer and Lev-Tov, 1997).

\section{Results}

Activation of the lamprey spinal locomotor networks was induced in two ways, (1) by superfusion with $150 \mu \mathrm{M}$ NMDA or (2) by electrical stimulation of one side of the spinal cord (either in the intact, partially or fully hemisected preparation) according to Cangiano and Grillner (2003). In all cases, activity was recorded extracellularly from the ipsilateral and contralateral ventral roots and intracellularly from fMNs and mMNs.

Fin motoneuron activity in the intact and intermediate-lesioned spinal cord during NMDA induced fictive

\section{locomotion}

Suprathreshold activity of intact spinal cord motoneurons could be recorded at the ventral roots a few minutes after the onset of superfusion with NMDA, although it generally took $>20$ min to over an hour until a stable left-right alternating discharge pattern was established (Figs. 1, 2A) (Pérez et al., 2007). The swimming pattern frequency varied between 2 and $4 \mathrm{~Hz}$, a typical range for $150 \mu \mathrm{M}$ NMDA (Grillner et al., 1981; Brodin et al., 1985). Under these conditions fMNs always showed a clear out-of-phase activity with the activity of the ipsilateral mMNs (Figs. 1, 2 A) (Shupliakov et al., 1992; Mentel et al., 2006). The membrane potential of fMNs is modulated by inhibitory synaptic inputs during ipsilateral mMN activity and excitatory synaptic inputs during contralateral mMN activity (Mentel et al., 2006).

Because intrasegmental and short range intersegmental contralateral signals (up to three segments) are not necessary to establish the phasing in activity between mMNs and fMNs (see Introduction) the question arose if contralateral signals play any role for the coordination between $\mathrm{mMN}$ and $\mathrm{fMN}$ activity. The relevant circuitry could, in principle, be located on the ipsilateral side. This question was studied by progressively increasing longitudinal midline lesions uniformly distributed along the entire piece of spinal cord, thus applying an approach similar to that used by Cangiano and Grillner (2003). One cannot assume that the commissural fibers involved in $\mathrm{fMN}$ and $\mathrm{mMN}$ coordination are evenly distributed along the cord. The use of this lesioning technique ensures a percentage-wise equal reduction in contralateral influence across all segments of the preparation.

Superfusion of the of $30-50 \%$ perforated spinal cord with $150 \mu \mathrm{M}$ NMDA induced, in all cases ( $n=5$ in four animals), a fictive swimming motor pattern with clear left-right alternation between the ventral roots (Fig. $2 B$ ). In the example shown in Figure $2 B$ the cycle period during NMDA-induced fictive locomotion was 250-300 ms corresponding to a swimming frequency of 3-4 Hz. The waveform correlation of the rectified ventral root recording of the ipsilateral side with the intracellular recording of a $\mathrm{fMN}$, shows an anticorrelation with a negative central peak at around zero (Fig. $2 \mathrm{Bi}$ ). In the same recording the correlation of the $\mathrm{fMN}$ with the rectified ventral root recording of the contralateral side shows a positive correlation with a clear central peak at around zero (Fig. 2 Bii). This was true for all fMNs recorded $(n=5)$. In this condition fMN phasing was thus unaffected.

When the midline lesion was increased to $50-70 \%$, superfusion with $150 \mu \mathrm{M}$ NMDA still induced a fictive swimming motor pattern with left-right alternation in all cases. However, in this situation fMNs no longer showed a membrane potential oscillation pattern that was clearly out-of-phase with the activity recorded at the ipsilateral ventral root (Fig. 2C) $(n=5$ in three animals). The clear hyperpolarization in the $\mathrm{fMN}$ associated with each activity burst recorded at the ipsilateral ventral root (Fig. 2A,B) (Mentel et al., 2006) was no longer detectable, but instead a depolarization was observed that was linked to the ipsilateral ventral root activity (Fig. 2C). For a 


\section{A intact spinal cord}

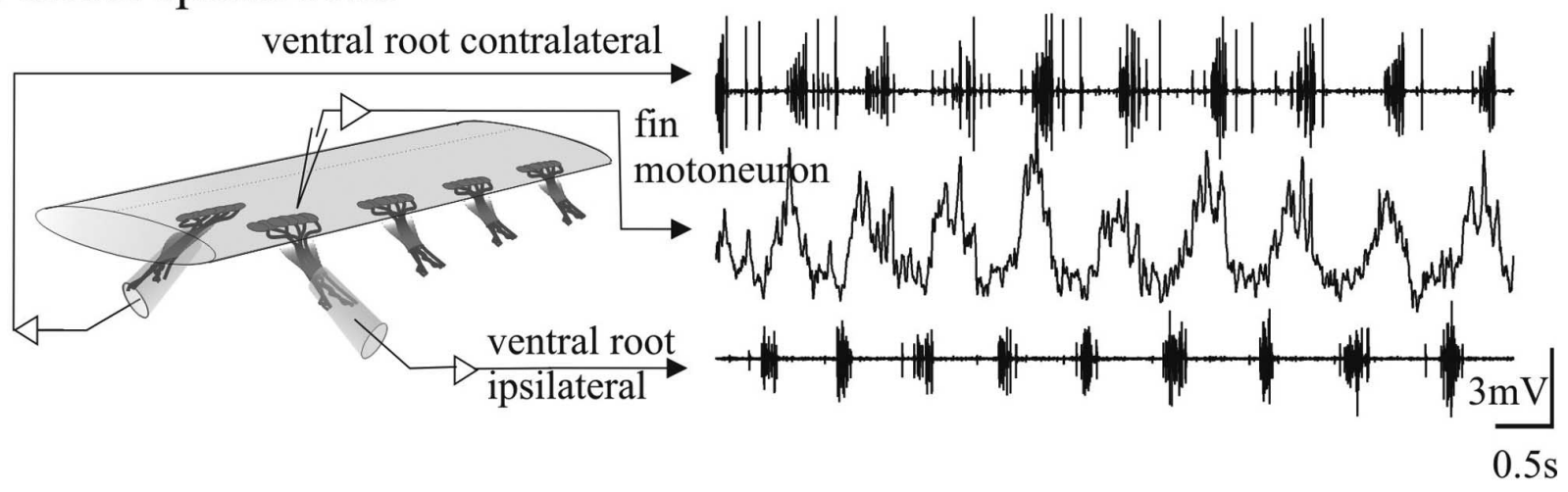

B $30 \%-50 \%$ of midline perforated $\mathbf{B i}$

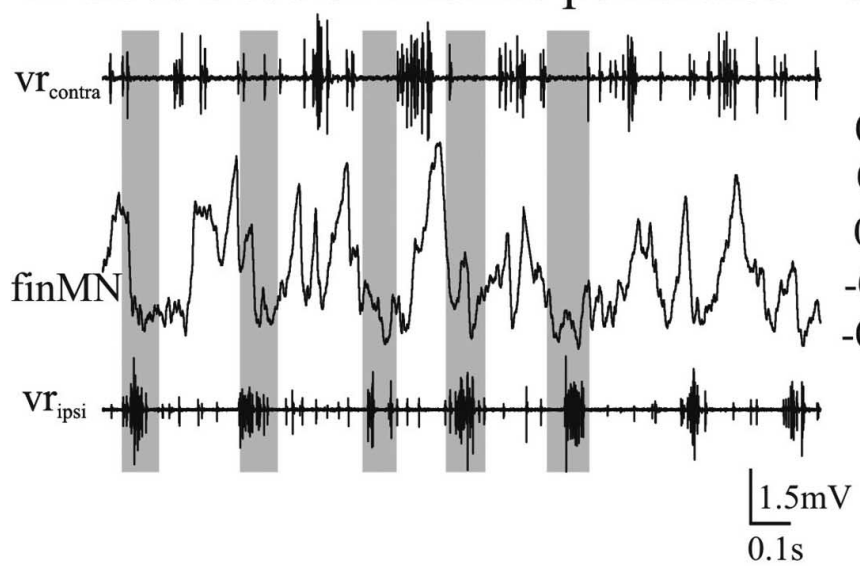

C $50 \%-70 \%$ of midline perforated

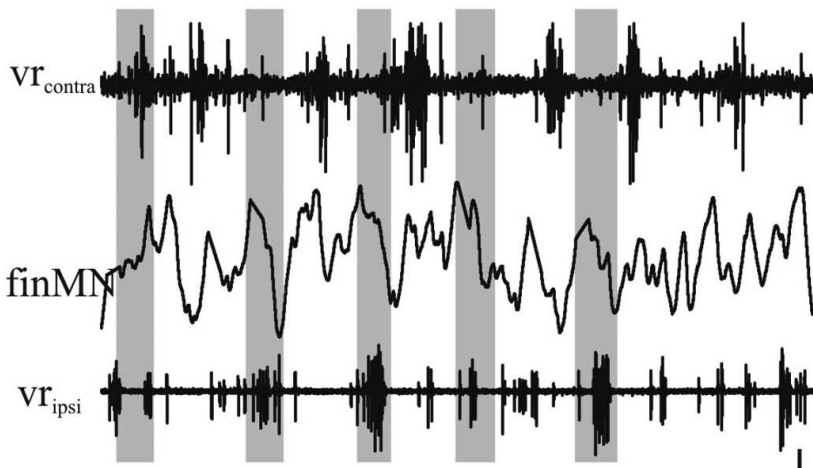

$\frac{3 \mathrm{mV}}{0.1 \mathrm{~s}}$
Bii
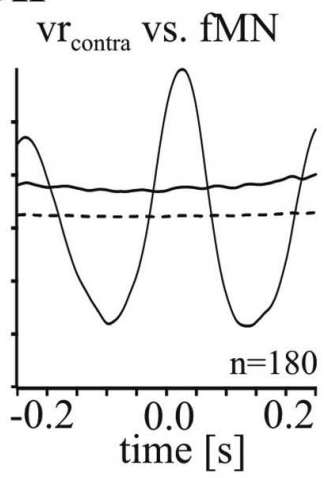

Cii

$\mathbf{C i}$ $\mathrm{vr}_{\mathrm{ipsi}}$ vs. fMN
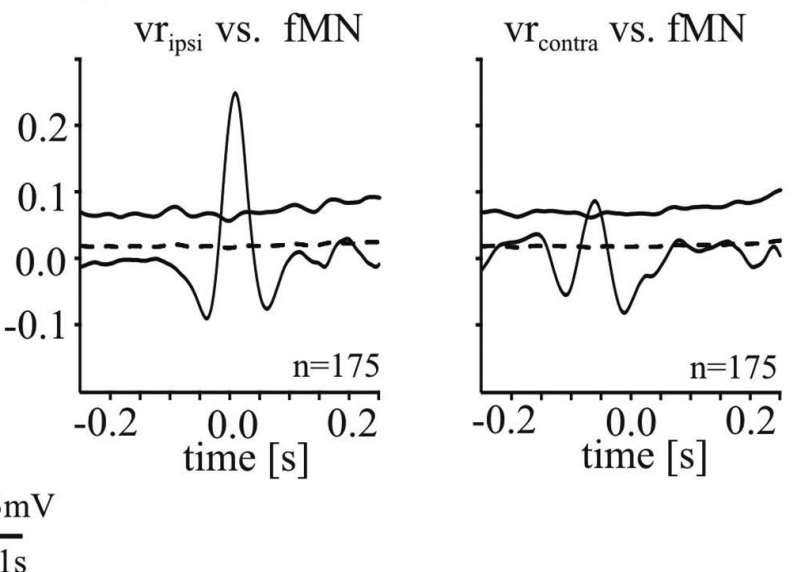

Figure 2. Fin motoneuron activity during NMDA-induced fictive swimming. $A-C$, Recording of the membrane potential oscillation of a fin motoneuron together with the ipsilateral and contralateral ventral root in an intact spinal cord $(\boldsymbol{A})$ and with intermediate midline lesions $(\boldsymbol{B}, \boldsymbol{C})$. In all experimental situations, an alternating activity pattern of the left and right side is evoked by superfusion of $150 \mu \mathrm{M}$ NMDA. $A$, Recording of the membrane potential oscillation of a fin motoneuron in an intact spinal cord. Fin motoneurons show a clear out-of-phase activity with the ventral root discharge of the same side. $\boldsymbol{B}$, When the midline of the spinal cord is lesioned $30-50 \%$, clear out-of-phase activity of fin motoneurons with the ipsilateral ventral root activity persists. $\boldsymbol{B i}$, The mean waveform correlation of the cell shown in B with the rectified ipsilateral ventral root activity shows a clear negative peak around zero and therefore an out-of-phase activity. Here and in all subsequent figures, the dotted horizontal line close to zero denotes the SE, whereas the solid horizontal line denotes four times SE (see Materials and Methods). Bii, The mean waveform correlation with the contralateral ventral root discharge shows a clear positive peak around zero and therefore an in phase activity of the fin motoneuron. $C$, When the midline of the spinal cord is perforated $50-70 \%$, fin motoneurons lose their out-of-phase activity and show instead an in-phase activity with the ipsilateral ventral root burst. The mean waveform correlation of the intracellular recording of the same cell with the rectified ventral root recording of the ipsilateral side shows a positive peak at zero (Ci) and no or a very weak negative correlation with the contralateral side (Cii).

quantitative comparison, waveform correlations were performed as described above (Fig. 2Ci,Cii). The waveform correlation of the intracellular recording with the ipsilateral rectified ventral root recording always showed a positive correlation (Fig. 2Ci), whereas the correlation with the con- tralateral side showed no or a weak negative correlation (Fig. 2 Cii). Thus, with $50-70 \%$ of the midline perforated, the membrane potential in fMNs was primarily affected by depolarizing synaptic drive which was in-phase with the activity of mMNs of the ipsilateral side (Fig. 2Ci). 
Phasing of fin motoneuron membrane potential oscillations during bouts of electrically evoked rhythmic bursting in the ipsilateral hemicord

From the experiments described above, it is clear that fMNs lose their out-of-phase coordination with ipsilateral mMNs, when the input from contralateral commissural interneurons is experimentally reduced below a certain level, although bilaterally alternating activity of mMNs persists. However, the fact that, after removal of $50-70 \%$ of the input of crossing fibers to fMNs, an in-phase depolarization with the ipsilateral mMNs became apparent indicates that fMNs might share common premotor elements with ipsilateral mMNs.

The change in the coordination of $\mathrm{fMN}$ activity was investigated by recording from fMNs in three experimental situations: (1) electrically induced unilateral locomotor activity in the intact spinal cord, (2) electrically induced unilateral locomotor activity released in the cord with partial midline lesions and (3) electrically induced unilateral locomotor activity released in the hemicord (cf. Fagerstedt et al., 2000; Cangiano and Grillner, 2003). As reported previously the release of locomotor bouts in the hemisected spinal cord represented an all or nothing phenomenon (Cangiano and Grillner, 2005). Therefore, brief pulse trains were applied on the lateral tract and their amplitude was progressively increased to a point when bouts of locomotor activity were reliably released (for details, see Materials and Methods).

Unilateral stimulation of the lateral tract of either side of the intact spinal cord elicited brief bouts of rhythmic activity on the side of stimulation. We never observed activity in the ventral roots of the contralateral side of the same segment (Fig. $3 A, A i$ ). Bout duration was much shorter $(<15 \mathrm{~s})$ than that reported for the hemicord or the partially lesioned cord (Cangiano and Grillner, 2003) (see below). For example, in a given preparation, with a sample of 9 stimulations, bout durations ranged from $3.2 \mathrm{~s}$ to $5.0 \mathrm{~s}$, being on average $4.2 \pm 0.58 \mathrm{~s}$. With this type of unilateral activation, fMNs exhibited rhythmic modulation in membrane potential on top of a tonic depolarization. The maximum depolarization of up to $5 \mathrm{mV}$ generally occurred at the beginning of a bout of activity and then slowly decayed to the level of the fMN resting membrane potential (Fig. 3A). The same unilateral stimulation applied to the hemi spinal cord or with partial lesions (see below) elicited a background depolarization that varied between 1 and $15 \mathrm{mV}$. During bouts of such unilateral activity in the
A intact spinal cord

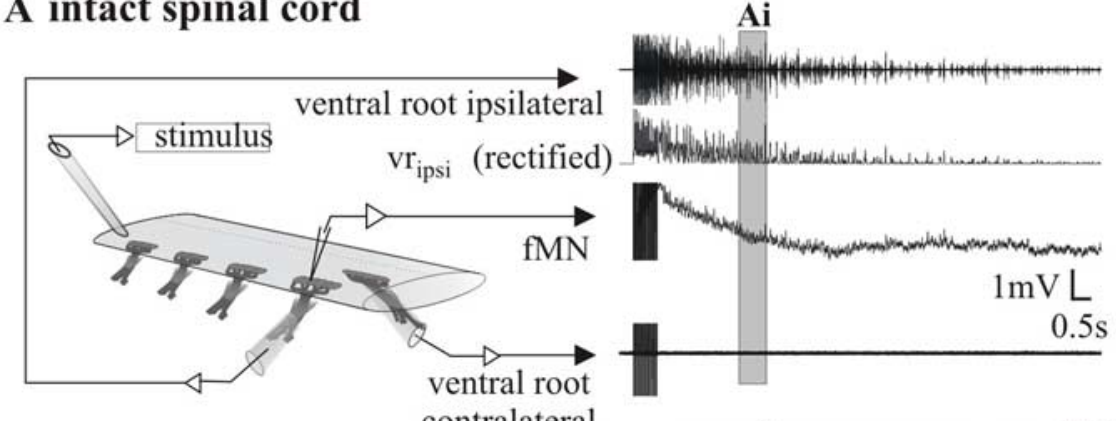

contralateral

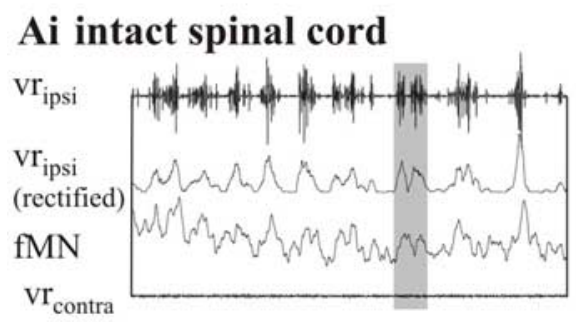

B $30 \%-50 \%$ of midline lesion

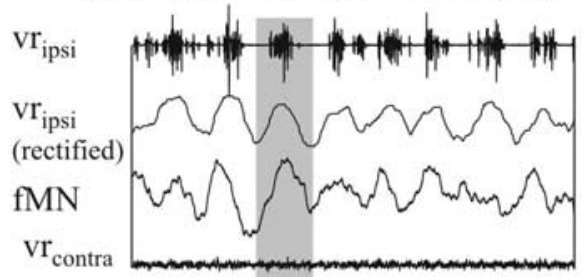

C $\mathbf{5 0} \% \mathbf{- 7 0} \%$ of midline lesion

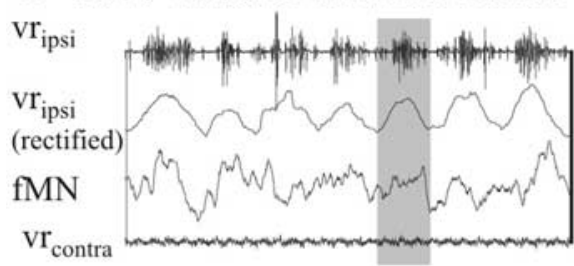

$1 \mathrm{mV}$

$\mathrm{Bi}$

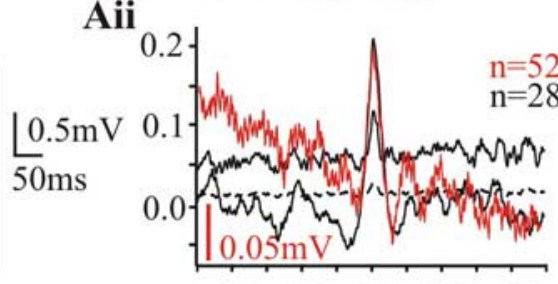

waveformcorr. $\mathbf{v r}_{\text {ipsi }}$ vs. fMN waveformavg. $\mathrm{vr}_{\mathrm{ipsi}} \mathrm{vs}$. fMN

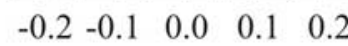

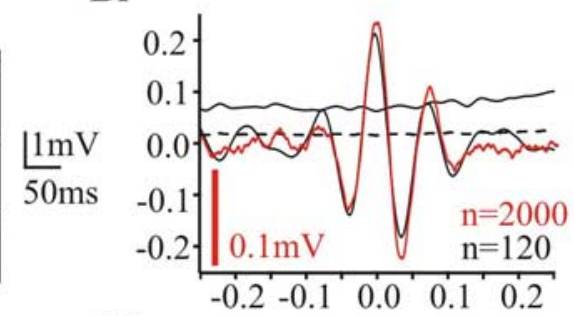

$\mathbf{C i}$
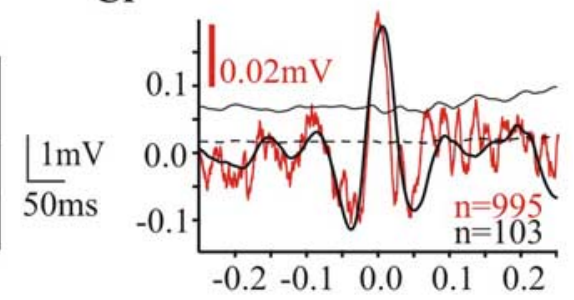

\section{D hemispinal cord}

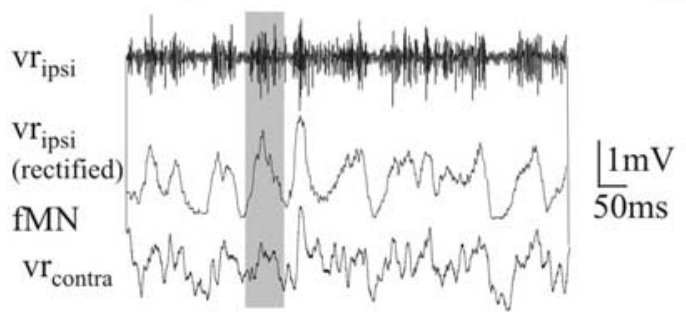

Di

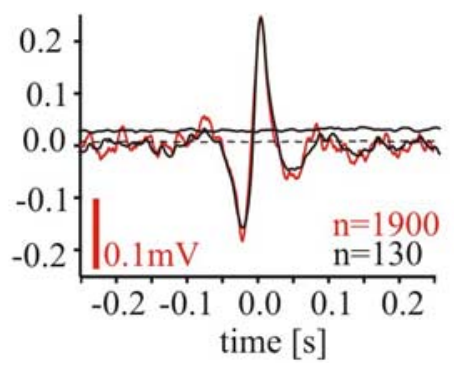

Figure 3. Intracellular recordings of fin motoneurons in an intact cord, a spinal cord with intermediate lesions ( $50-70 \%$ and $30-50 \%$ of midline perforated), and a fully hemisected cord, during locomotor burst activity evoked by unilateral electrical stimulation. $\boldsymbol{A}$, Bout of self-sustained activity in an intact cord evoked by electrical stimulation. $\boldsymbol{A i}, \boldsymbol{B}, \boldsymbol{C}, \boldsymbol{D}$, Top trace, Recording from ipsilateral ventral root $\left(\mathrm{vr}_{\mathrm{ipsi}}\right)$; second trace from top, trace giving the rectified and integrated ipsilateral ventral root activity $\left(\mathrm{vr}_{\mathrm{ipsi}}\right.$, rectified); third trace from top, intracellular recording from fMN; bottom trace, recording from contralateral ventral root $\left(\mathrm{vr}_{\text {contra }}\right)$. In the intact cord $(\boldsymbol{A} \boldsymbol{i})$, as well as in spinal cords with intermediate lesions (30-50\% in $\boldsymbol{B}$ and $50-70 \%$ in $\boldsymbol{C}$ ) and the hemicord (D), fin motoneurons receive an in-phase drive when activity bouts are triggered by electrical stimulation. $\mathbf{A i i ,} \mathbf{B i}, \mathbf{C i}, \mathbf{D i}$, Waveform correlations (black traces) of the membrane potential of fin motoneurons and the rectified ventral root recording in all experimental situations are positively correlated at around zero indicating a drive in-phase with the ventral root discharge. In all correlations dashed lines mark the SE and solid lines mark the fourfold SE. Red traces show the mean waveform averages based on the mid-burst of the ipsilateral ventral root burst. In all cases, the time course of the average is close to identical with the course of the correlations indicating clearly an in phase drive to fin motoneurons during the ipsilateral bursting activity. 
intact cord patterning of fMN membrane potential was evident $(n=4)$ and were found to be modulated in phase with the (ipsilateral) ventral root activity (Fig. 3Ai,Aii). The average of multiple sweeps of the intracellular recording triggered by the midburst of the ipsilateral ventral root burst showed a clear in-phase excitation in $\mathrm{AMNns}$ (Fig. 3 Aii, red trace). Mean waveform correlations performed by correlating consecutive stretches of $0.5 \mathrm{~s}$ length of the intracellular recording and the rectified ventral root recording showed the same time course compared with the waveform average, with a positive peak at around zero (Fig. 3Aii, black trace). To better assess the significance of the observed correlation coefficient, both the SE (dashed line in all correlations) and the fourfold SE (solid line in all correlations) were calculated and shown in the figures (cf. Kremer and Lev-Tov, 1997; Cangiano and Grillner, 2005).

Similarly, after partial lesioning in the range of $30-50 \%$ and $50-70 \%$ it was possible to evoke unilateral activity bouts by stimulating the lateral tract of either side of the spinal cord (Fig. $3 B, C)$. fMN activity was in-phase with the ipsilateral ventral root activity after midline lesioning of $30-50 \%$ (Fig. $3 B, B i$ ). The bouts released after electrical stimulation were significantly longer than in the intact cord (i.e., 37 to 265 s, with a mean of $107.0 \pm 68.3 \mathrm{~s}$ in $n=23$ bouts in four animals). In preparations with lesions of $50-70 \%$, all recorded fMNs $(n=5$ in three animals) also displayed a rhythmic synaptic drive which was in phase with the ipsilateral ventral root bursts on the stimulated side (Fig. $3 C)$. Calculation of waveform averages of the recording of a $\mathrm{fMN}$ triggered at the mid-burst of the corresponding ipsilateral ventral root discharge (Fig. 3Ci, red trace), as well as waveform correlations (Fig. 3Ci, black trace) showed an in-phase modulation of fMNs under these conditions. The mean duration of bouts elicited with electrical stimulation was similar to that with $30-50 \%$ lesioning, being $115.6 \pm 86.52 \mathrm{~s}(n=17$ bouts in three animals, range $8.0-374.0 \mathrm{~s})$.

In the hemicord (i.e., after a complete midline section), the activity bouts had a mean duration of $117.8 \pm 44.35 \mathrm{~s}(n=33$ bouts in four animals), with a minimum of $30 \mathrm{~s}$ and maximum of $234 \mathrm{~s}$ being observed. The mean cycle period of the rhythmic bursting in the preparation shown in Figure $3 D$ was $74.9 \pm 48.7$ $\mathrm{ms}(n=1496)$. The average cycle period ranged from 71.4 to $104.3 \mathrm{~ms}$ in the four preparations tested. It is thus in the same range as described by Cangiano and Grillner (2005) for hemicord rhythmic bursting activity. In the hemicord all fMNs $(n=6$ in four preparations) displayed membrane potential oscillations, which were modulated in-phase with the ipsilateral segmental ventral root activity (Fig. 3D). The average of multiple sweeps of the intracellular recording triggered by the mid-burst of the ipsilateral ventral root again showed a clear in-phase excitation in fMNs (Fig. 3Di, red trace). The mean waveform correlations showed the same time course as the waveform average, with a positive peak at around zero (Fig. 3Di, black trace). The average amplitude of the membrane potential oscillations ranged from 0.05 to $0.26 \mathrm{mV}$ for the individual recorded $\mathrm{fMN}$ and was on average $0.17 \pm 0.09 \mathrm{mV}$. Recordings from mMNs $(n=2)$ showed a similar membrane potential time course, waveform average and waveform correlation phasing (data not shown) (cf. Cangiano and Grillner, 2005).

From the above it appears that $\mathrm{fMNs}$ receive common synaptic inputs from the same ipsilateral rhythmic premotor networks as mMNs. The fact that these common ipsilateral inputs only become evident (1) after removal of the synaptic input from contralateral crossing fibers (see above) or (2) after selective activation of the ipsilateral hemicord networks, indicates that they are normally masked when contralateral activity and inputs are present. To directly compare the synaptic drive converging onto fMNs and mMNs within their hemicord, both types of motoneurons were recorded simultaneously in four experiments. In these experiments the spinal cord was lesioned $30-50 \%$ and unilateral electrical stimulation was applied. Two motoneurons, one from each functional population in the same or neighboring segments were impaled with sharp electrodes (Fig. 4). For a morphological identification the fMNs were prelabeled with FDA as described in the Materials and Methods. Both prelabeled fMNs and nonlabeled mMNs were injected with RDA (Fig. $4 E$ ). Under these conditions the membrane potential modulation of the $\mathrm{fMN}$ and the mMN showed a very similar, although not identical, pattern (Fig. 4Ai). A strong positive correlation was found between the membrane potential oscillations of the $\mathrm{fMN}$ and that of the $\mathrm{mMN}$ (Fig. $4 B$ ), and with the rectified ipsilateral ventral root recording in the same segment (Fig. 4C,D). The fact that the correlation coefficient between $\mathrm{fMN}$ and $\mathrm{mMN}$ is high indicates that the two cell types receive a common synaptic drive from the premotor network. These data from electrical stimulation further support the conclusion made under NMDA superfusion that fMNs and $\mathrm{mMN}$ receive a common excitatory synaptic drive from the ipsilateral spinal locomotor network.

\section{The role of intersegmental contralateral synaptic inputs for coordinating $\mathrm{mMN}$ and $\mathrm{fMN}$ during locomotion}

The conditions under which the alternating $\mathrm{AMN}$ and $\mathrm{mMN}$ pattern was retained, were examined in a preparation with a local hemisegment receiving contralateral inputs arising only from six to eight segments rostral from it. To do so, a long piece of the spinal cord from the fin region was lesioned sagittally up to eight segments from the caudal recording site, leaving only a few segments of its rostral portion intact (between three and six) (Fig. $5 A$ ). Fictive locomotion was induced by bath application of NMDA and the activity of the spinal cord was recorded from opposite ventral roots in the intact rostral part, as well as from the hemisegment of the recording site in the caudal part (Fig. $5 B$ ). Under these conditions fMNs retained a modulation out-ofphase with the segmental ipsilateral ventral root discharge as well as the rostral ipsilateral ventral root discharge $(n=5)$ (Fig. $5 C, D)$. It is important to note that slight deviations from a complete out-of phase modulation for the correlations resulted from the 8 segments located between the fMNs and the rostral ventral root (Fig. 5D), as well as a rather spread-out activity in the caudal segment recorded (Fig. $5 C$ ). Although the experiments described in previous sections indicated that contralateral activity is necessary to establish out-of-phase coordination of fMN and $\mathrm{mMN}$ on the ipsilateral side, these latter data showed that rostrally originating intersegmental contralateral signals are in fact sufficient for this to occur.

In the lamprey spinal cord excitatory commissural interneurons with long descending axons have been described [excitatory contralaterally and caudally projecting interneurons (CC-E)] (Buchanan, 1982). To investigate whether a direct monosynaptic connection from these CC-E interneurons might be responsible for driving fMNs, the axons of these long range interneurons were stimulated contralaterally. An extracellular electrode was positioned on the contralateral side of the midline (Fig. 6A) (Alford and Grillner, 1991), where it is known that these interneurons' axons pass superficially through the ventral commissure. FMNs were identified by double-labeling (see Materials and Methods) and by the orthodromic action potential recorded at the ventral root (Fig. 6 Bii). After compound electrical stimula- 
A

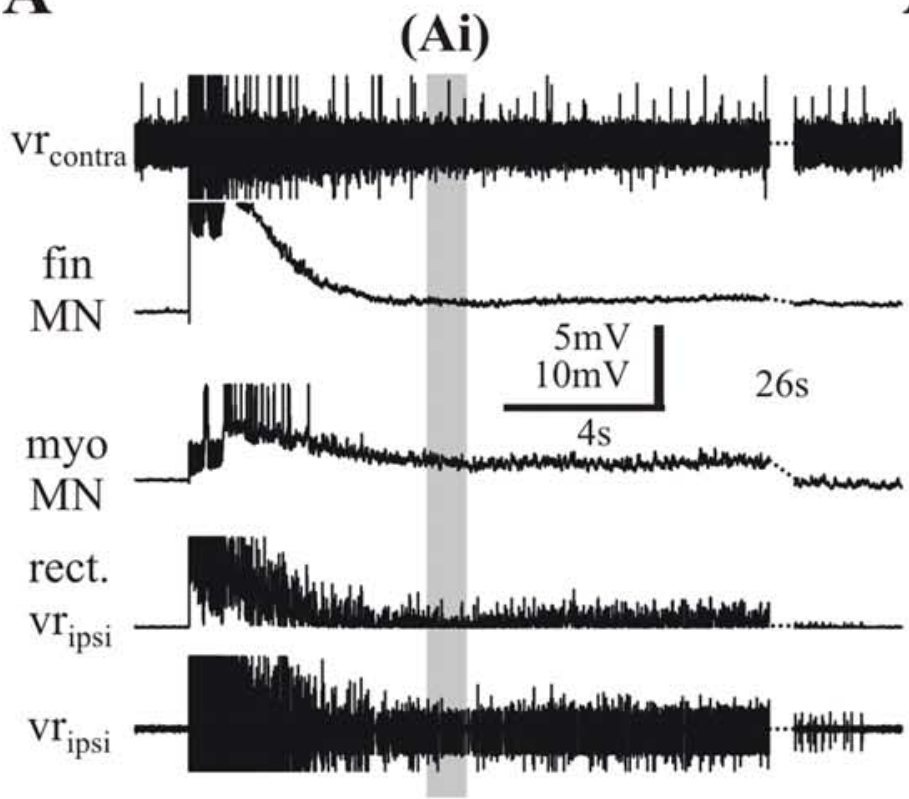

Ai
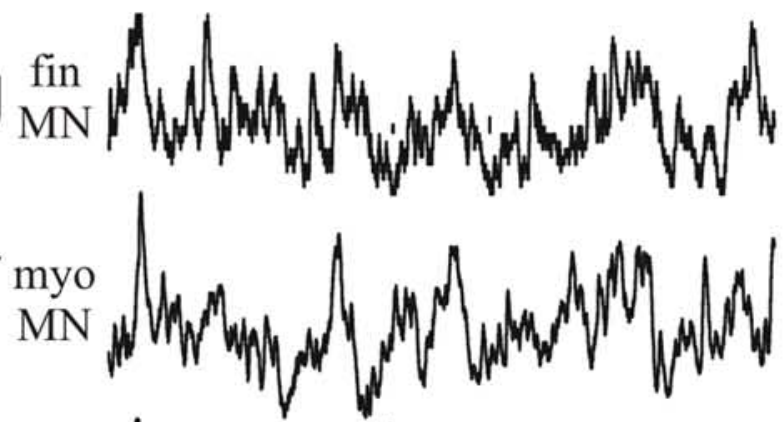

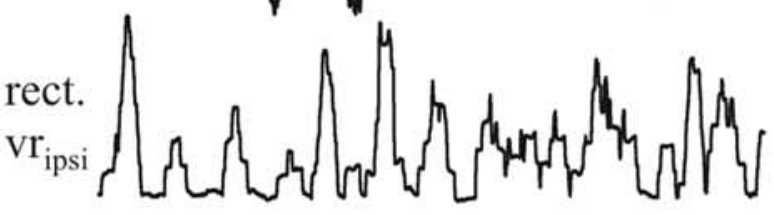

$0.5 \mathrm{mV}$

$2.5 \mathrm{mV}$

$0.25 \mathrm{~s}$
B

fMN vs. myoMN

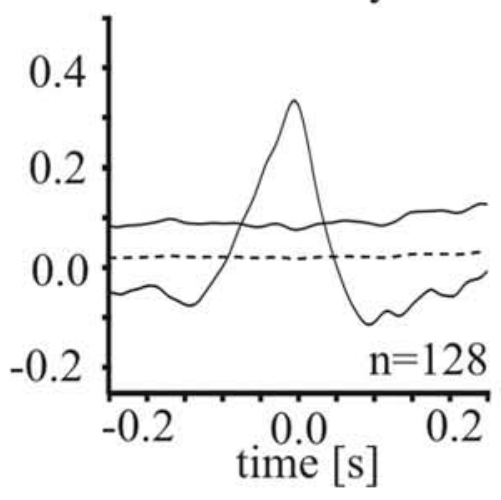

C

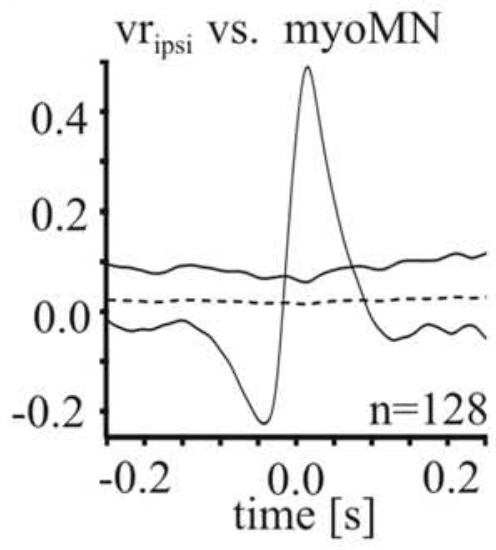

D

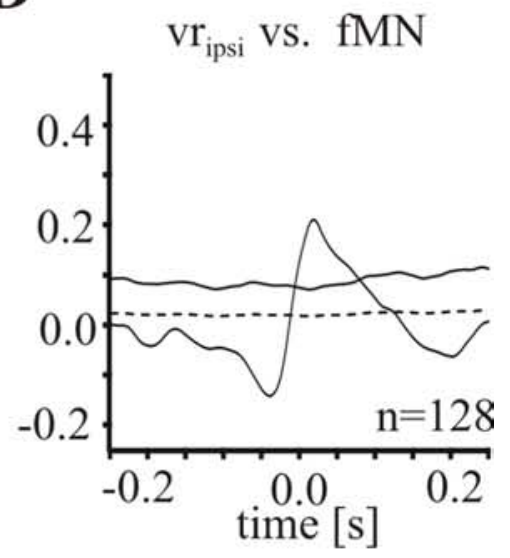

E

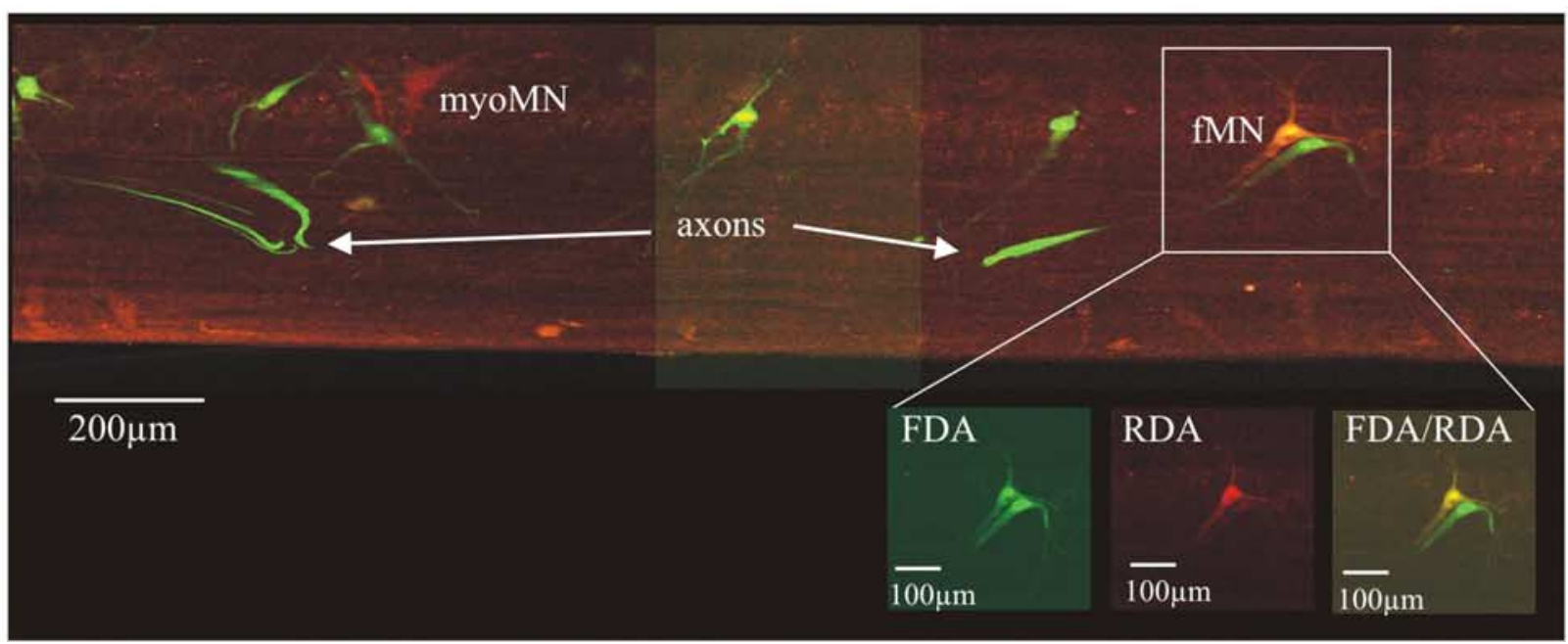

Figure 4. Simultaneous recordings of a fin motoneuron and a myotomal motoneuron during electrically evoked motor burst activity in a piece of spinal cord lesioned 30 - $50 \%$ and electrically stimulated on the ipsilateral side. $A$, One sequence of a bout of activity. Top trace, Activity of the contralateral ventral root ( $\mathrm{vr}_{\text {contral }}$; second and third traces, intracellular recordings from a fMN and a mMN; fourth trace, rectified ipsilateral ventral root recording; fifth trace, activity of the ipsilateral ventral root $\left(\mathrm{vr}_{\mathrm{ipss}}\right)$. Toward the end, $26 \mathrm{~s}$ are cut out (stippled (Figure legend continues.) 
A B
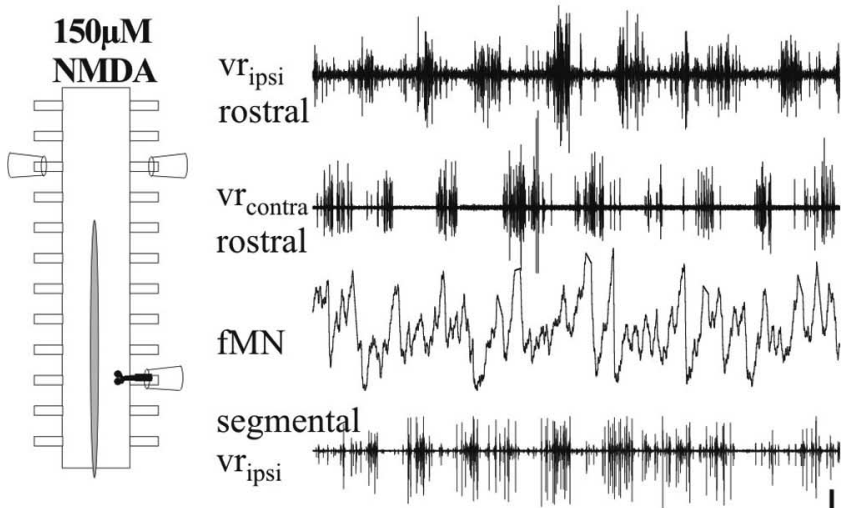

$5 \mathrm{mV}$

C

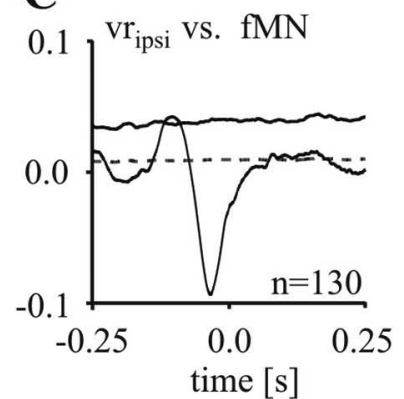

D

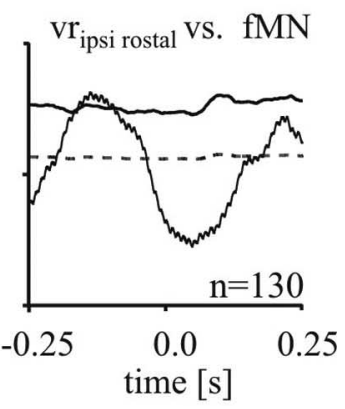

Figure 5. Persistence of contralateral inputs to fMNs in the partially hemisected cord. $\boldsymbol{A}$, In this experiment, a sagittal lesion extends from a region five segments rostral to the recording site (black MN outlines) down to the caudal end of the cord. $\boldsymbol{B}$, Under these conditions, the ventral root discharge in the segment where the fMN is recorded (segmental $\mathrm{vr}_{\mathrm{ipsi}}$ ) shows weak phasic activity, although in comparison with the other ventral root recordings a rhythmic burst pattern is still detectable. $\boldsymbol{C}, \boldsymbol{D}$, Waveform correlations of the fMN with the rectified recordings of the segmental ipsilateral ventral root $(C)$ and the ipsilateral ventral root of the first or second intact segment rostral to the recording site (D) shows a negative peak around zero indicating an out-of-phase drive to fMNs. Please note that although the rhythmic discharge pattern recorded at the segmental ventral root seems to be less well structured a clear negative peak is detectable, indicating a persistent out-of-phase drive to fMNs.

tion of axons arising from contralateral intersegmental interneurons, all recorded fMNs received short latency EPSPs ( $n=5$ in five preparations) (Fig. 6 $\mathrm{B}, \mathrm{Bi}$ ), which reliably followed a one-to-one stimulation-response up to $10 \mathrm{~Hz}$. Importantly, in two of these experiments, we recorded subsequently from a mMN in the same segment close to the previously recorded $\mathrm{fMN}$. When applying the same stimulus with the same stimulation strength only a compound IPSP was recorded (Fig. 6C,Ci), exemplifying the well known inhibitory synaptic connection of commissural interneurons to mMNs, as reported previously with this same type of experimental set-up (Alford and Grillner, 1991; Büschges et al., 2000).

\footnotetext{
(Figure legend continued.) lines) to increase the region of presentation. The gray bar indicates the episode of the recording shown in $\mathbf{A i}$ in a higher time resolution without top and bottom trace. The fin motoneuron as well as the myotomal motoneuron receives excitatory synaptic input in-phase with the activity of the ipsilateral ventral root. $\boldsymbol{B}$, Waveform correlations between the two motoneurons show a positive peak around zero. $C, D$, Waveform correlations of each motoneuron with the rectified ipsilateral ventral root recording show positive peaks around zero. $\boldsymbol{E}$, Recorded motoneurons were filled with RDA (red marker) via the electrode. Fin motoneurons were retrogradely prelabeled with FDA (green marker). The yellow cell (fMN) is a double-labeled fin motoneuron (see inset).
}

Together with the results of Mentel et al. (2006) the present findings indicate that intersegmental contralateral inputs might be necessary for generating out-of-phase modulation of membrane potential in $\mathrm{fMNs}$ during fictive locomotion in the intact cord. To test the sufficiency of intrasegmental contralateral inputs to fMNs, we recorded fMNs in an experimental situation that excluded the influence of long range intersegmental activity. Two contralateral segments were left attached in the middle region of a 20 segment long piece of an otherwise hemisected spinal cord and the spinal locomotor network was activated with NMDA (Fig. 7A). Three fMNs recorded at the level of the contralateral segments showed under these conditions in-phase modulation with the ipsilateral segmental ventral root. Figure $7 B$ shows the activity of one of these fMNs together with that in the ipsilateral ventral root. Their waveform correlation is shown in Figure $7 C$. This result further substantiates the significance of intersegmental contralateral inputs to $\mathrm{fMNs}$, in the generation of their out-of-phase activity.

\section{Fin motoneuron activity at onset of fictive locomotion after superfusion with NMDA}

When NMDA is applied to the spinal cord it can take more than an hour until the fictive swimming pattern is fully and robustly established (Brodin et al., 1985; Pérez et al., 2007). Often motor activity in the spinal cord begins with periods of asymmetric activity, with one side prevailing over the other. The initially unorganized discharge pattern of motoneurons monitored in the ventral root then slowly develops into a regular alternating rhythmic burst pattern between both sides of the spinal cord. We studied the modulation of membrane potential in $\mathrm{fMNs}$ during this early phase of establishing the fictive swimming pattern $(n=4$ in four animals). We observed in all recordings that shortly after the start of superfusion with NMDA fMNs received a drive in-phase with the ipsilateral segmental ventral root (Fig. $8 A-D$ ). As the normal organization of the motor pattern between the two sides progressively developed, the clear out-of-phase correlation to mMNs became apparent (Figs. 1, 2A, 8E) (Mentel et al., 2006). This observation is further exemplified by comparing the waveform correlation between the fMN and the ipsilateral ventral root with the waveform correlation between both ventral root recordings (Fig. 8, compare $A-D, E$ ). In-phase modulation often outlasted the early onset of fictive locomotion, where the ventral roots display alternating but not fully established motor activity (Fig. $8 B-D$ ). These results therefore support our conclusion that ipsilateral elements of the swimming pattern generating network have direct access to fMNs. It can further be concluded that only when there is a stable rhythm established between the two sides of the spinal cord, fMNs are driven in a reciprocal relationship with the ipsilateral mMNs in fictive locomotion.

\section{Discussion}

In the present study (1) we show that, during symmetric locomotor activity, the functionally appropriate phasing between ipsilateral pools of motoneurons (i.e., fMNs and mMNs) depends on the activity of intersegmental crossing fibers; (2) we provide evidence that $\mathrm{fMNs}$, in contrast to $\mathrm{mMNs}$, receive excitatory synaptic inputs from intersegmental commissural interneurons; and (3) we show that different ipsilateral motoneuron pools ( $\mathrm{fMN}$, $\mathrm{mMN}$ ) receive common rhythmic synaptic drive from the ipsilateral premotor network that can be unmasked in vitro under particular experimental conditions (cord lesions or unilateral electrical stimulation) or during the initial phases of fictive locomotion. 
Coordination of activity between mMNs and $\mathrm{fMNs}$ in the spinal cord

During locomotion, both in vivo and in vitro, fin muscles and $\mathrm{fMNs}$ exhibit activity out-of-phase to that of ipsilateral myotomal muscles and mMNs (Mentel et al., 2006). How is the $\mathrm{fMN}$ activity coupled to the activity of the mMNs? Previous studies have shown that a morphological subpopulation of fMNs has dendritic arborizations crossing the midline under and/or above the central canal (Shupliakov et al., 1992). This specific feature initially gave rise to the question as to what role intrasegmental contralateral inputs may play in phasing the $\mathrm{MN}$ activity. A midline lesion in the segment containing a $\mathrm{fMN}$, however, does not affect its normal phasing in the locomotor cycle (Mentel et al., 2006), indicating that intrasegmental synaptic inputs from the contralateral hemisegment to $\mathrm{fMNs}$ are not required. The potential relevance of such contralateral inputs comes from an investigation by Wallén et al. (1985), in which they reported that mMNs innervating the most dorsal or ventral trunk muscles have dendritic arborizations crossing the midline. These mMNs showed a slightly different phasing pattern when compared with mMNs of the same segment which lacked such dendritic arborizations. Specifically, they displayed a membrane potential oscillation with a depolarizing and hyperpolarizing phase up to one-fourth of a cycle earlier. A complete out-of-phase modulation was, however, not observed.

However, by increasing the extent of sagittal midline lesions, we have now shown that the coordination of activity between hemisegmental mMNs and fMNs in the locomotor cycle indeed depends on contralateral synaptic inputs. When the spinal cord midline was perforated $>50$ $70 \%$, fMNs were no longer driven out-of-phase with the mMNs, despite myotomal activity still alternating between left and right. Under these conditions an in-phase synaptic drive to fMNs was unmasked, indicating that contralateral signals are required to establish the normal coordination between fMNs and mMNs.

How could the out-of-phase coordination between mMNs and $\mathrm{fMNs}$ be mediated? The out-of-phase modulation of fMNs was maintained following a sagittal midline lesion of up to eight segments rostral to the recording site, indicating a contribution of long range intersegmental commissural fibers. Furthermore, removing contralateral intersegmental inputs to the recorded fMNs resulted in a loss of their out-of-phase modulation. We also show that $\mathrm{fMNs}$ receive monosynaptic EPSPs from commissural caudally projecting interneurons with axons crossing at least five segments rostral to the postsynaptic fMN. The specificity of such excitatory contralateral inputs for $\mathrm{fMNs}$ is substantiated by the observation that at the same stimulation site and stimulation strength, mMNs of the same segment were found to receive IPSPs from commissural interneurons of the contralateral side (Alford (data not shown)
B fin motoneuron

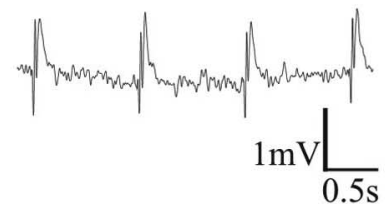

C myotomal motoneuron

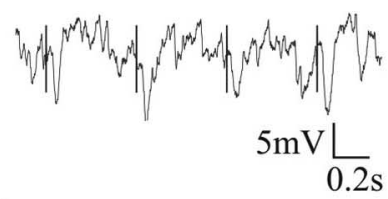

$\mathbf{B i}$

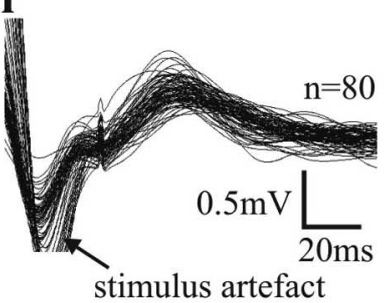

$\mathbf{C i}$
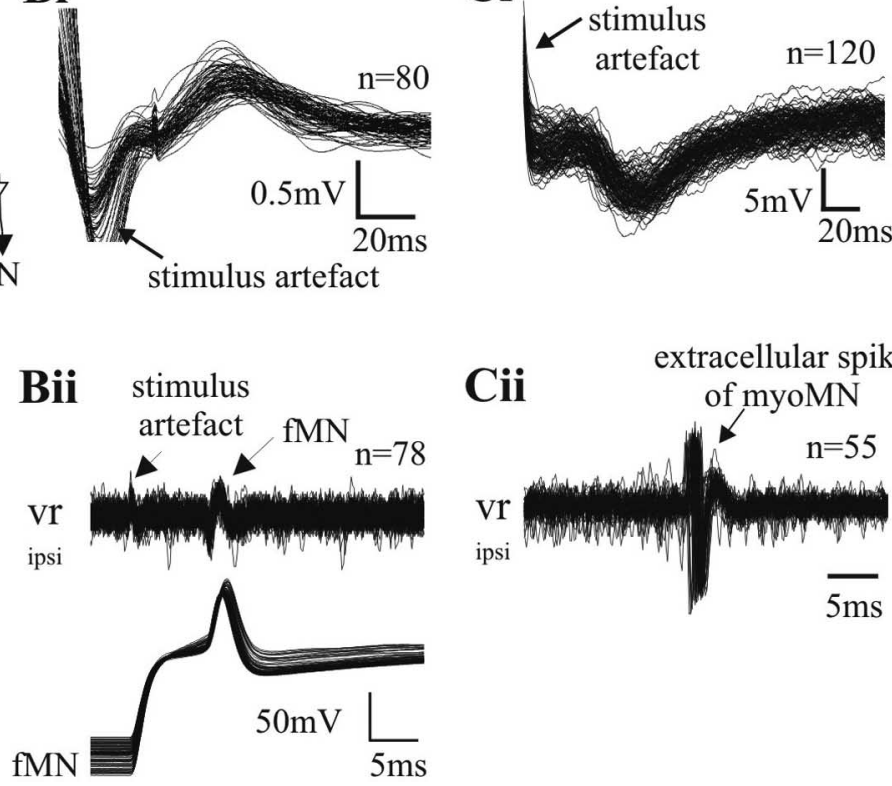

$5 \mathrm{~ms}$

Figure 6. Connectivity of commissural long-range projecting interneurons to fin and myotomal motoneurons. $\boldsymbol{A}$, Schematic drawing of the experimental set up for the stimulation experiments. The stimulus electrode is placed contralaterally eight stimulus artifact demonstrates the short, constant latency occurrence of the evoked EPSP, indicating a monosynaptic connection motoneuron, a dye was injected into the cell at the end of the experiment (data not shown). $\boldsymbol{C}$, The same stimulus strength (tested between 0.001 and $0.03 \mathrm{~mA}$ stimulation strength) at the same stimulus site evokes IPSPs in myotoma constant latency of the evoked IPSP between projecting axons and mMN. Cii, Identification of the cell shown in Ci as a motoneuinterneurons. To confirm the recording of a myotomal motoneuron, dye was injected into the cell at the end of the experiment

and Grillner, 1991; Büschges et al., 2000). Buchanan (1982) first identified intersegmentally caudally projecting commissural interneurons that monosynaptically excite motoneurons in the lamprey spinal cord, termed CC-E interneurons. Individual CC-E interneurons can show in-phase activity to the ipsilateral ventral root and, thus, with contralateral fMN (Buchanan, 1986). Together, these results strongly indicate that these commissural excitatory interneurons, first described by Buchanan (1982, 1986), are responsible for activating fMNs during fictive locomotion.

As an aid to a possible interpretation of our experiments the scheme in Figure 9 summarizes both current knowledge (gray elements and connections) about the spinal swimming networks driving $\mathrm{mMNs}$ and our new findings (black elements and connections) on the source of inputs to fMNs. In the intact spinal cord displaying regular alternating activity between $\mathrm{fMN}$ and $\mathrm{mMN}$, the excitation of $\mathrm{fMN}$ is most likely driven by commissural excitatory interneurons (Fig. 6) (see Discussion above), which are in turn driven by the contralateral excitatory kernel. In 
A

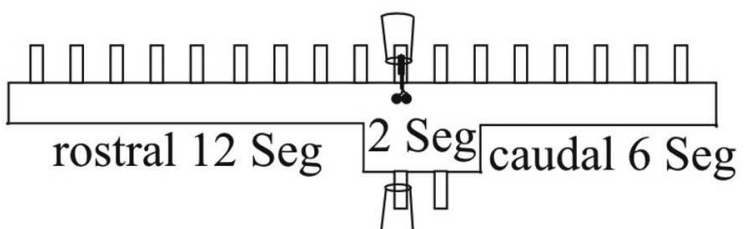

B

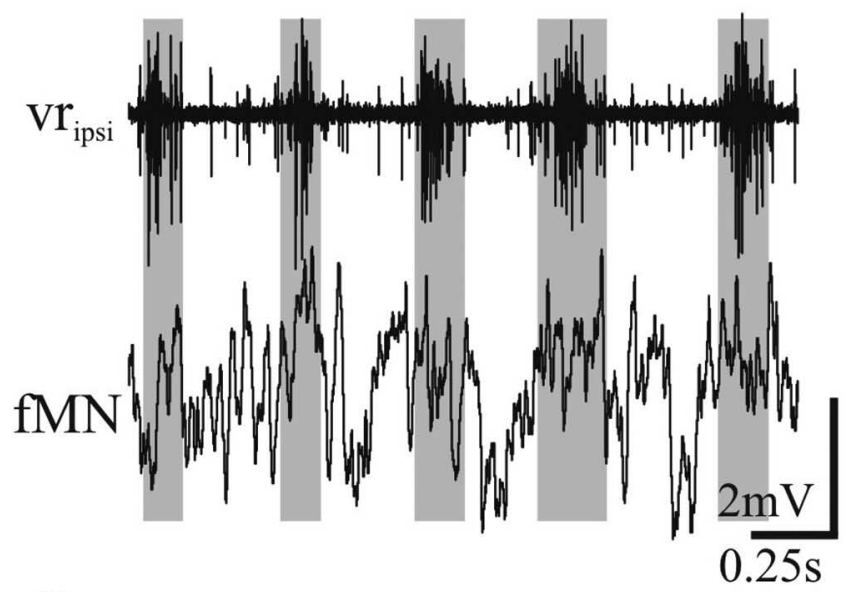

C

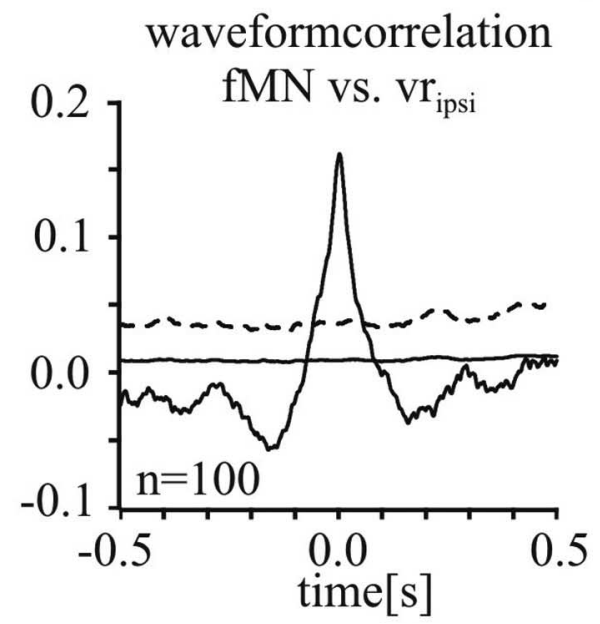

Figure 7. A, To investigate the phasing of fMN activity in the absence of long-range contralateral intersegmental drive, fictive locomotion was evoked with NMDA in a preparation which had all contralateral hemisegments removed except for two central ones. $\boldsymbol{B}$, Intracellular recording of a fMN located ipsilaterally in one of the two central segments. Note the in-phase modulation of the fMN membrane potential with the activity of the ipsilateral ventral root. $\boldsymbol{C}$, Waveform correlation between the membrane potential in the fMN and the rectified recording of the segmental ipsilateral ventral root. Differing from the other figures, the solid line marks the SE and the stippled line marks the fourfold SE.

antiphase with excitation, $\mathrm{fMN}$ also receive inhibitory input (Mentel et al., 2006), which is thus concurrent with the excitation of ipsilateral mMN. This may originate from the small ipsilateral inhibitory interneurons (Buchanan and Grillner, 1988) known to provide monosynaptic inhibition of motoneurons and to be active in the appropriate phase of the locomotor cycle [Fig. 9, gray shaded and stippled inhibitory interneurons (iINs)]. These neurons receive excitatory input from ipsilateral excitatory interneurons (EINs). In the hemicord the situation is the converse and fMNs instead receive a rhythmic synaptic drive in-phase with the mMNs (Buchanan and Grillner, 1987; Cangiano and Grillner, 2005), which is unmasked in the extensively transected cord or

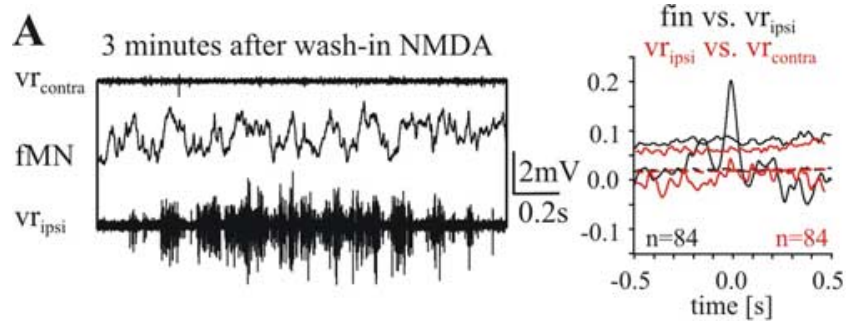

B 10 minutes after wash-in NMDA

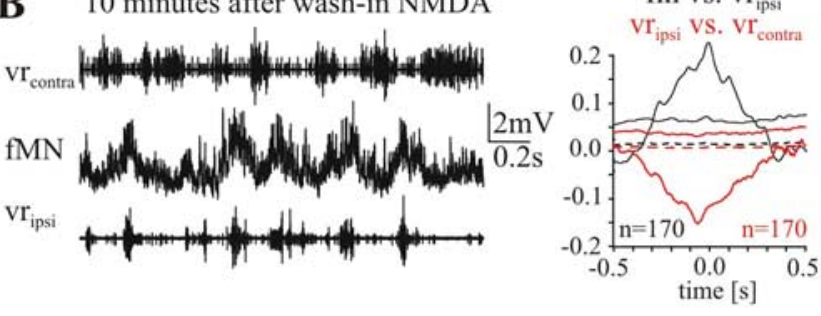

C 15 minutes after wash-in NMDA

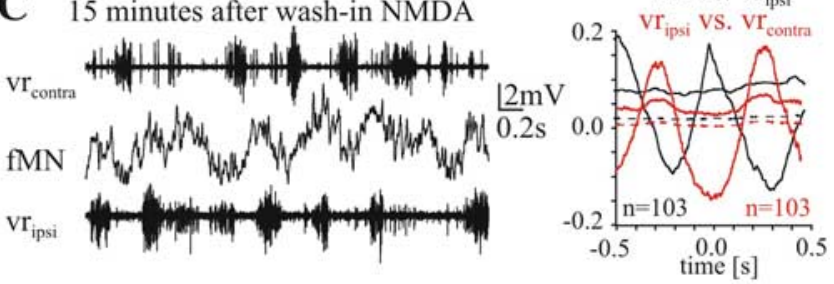

D 10 minutes after wash-in NMDA
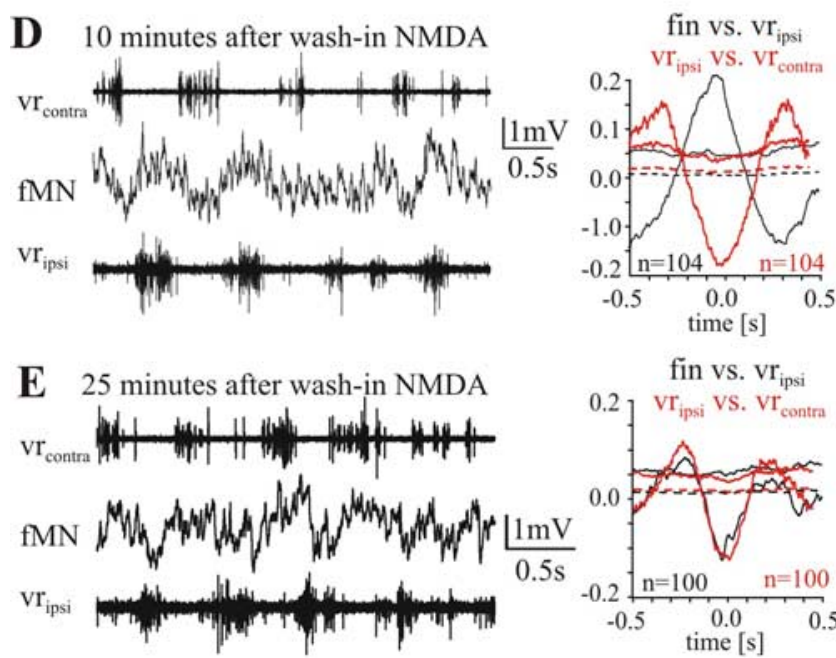

fin vs, vr

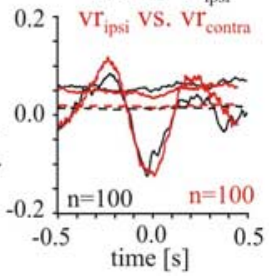

Figure 8. Activity of fin motoneurons at the onset of NMDA-induced fictive locomotion. $\boldsymbol{A}-\boldsymbol{D}$, Membrane potential modulation in four different $\mathrm{fMNs}$, respectively at $3(\boldsymbol{A}), 10(\boldsymbol{B}), 15$ (C), and 25 (D) min after the onset of superfusion with $150 \mu \mathrm{m} \mathrm{NMDA}$. On the right-hand side of each recording the waveform correlations are shown: the black curves belong to the correlation between $\mathrm{FMN}$ and the ipsilateral ventral root; the red curves belong to the correlation between the two contralateral ventral root recordings shown on the left. $\boldsymbol{D}, \boldsymbol{E}$, Membrane potential modulation of a fMN recorded at $10(\boldsymbol{D})$ then $25(\boldsymbol{E})$ min after NMDA application when the regular out-of-phase membrane potential modulation is established. Please note that during the rather irregular activity on both sides of the spinal cord occurring briefly after the start of NMDA superfusion, fMNs are depolarized in phase with the ipsilateral ventral root $(\boldsymbol{A}-\boldsymbol{D})$.

with unilateral electrical stimulation of the intact cord (Fig. 9, dashed lines from EINs to $\mathrm{fMN}$ ). The peak-to-peak amplitude of these in phase membrane potential oscillations in the hemicord was on average $0.17 \pm 0.09 \mathrm{mV}$, thus being significantly smaller than the amplitude reported for mMNs [3.05 $\pm 0.59 \mathrm{mV}$ for 14 mMNs (Cangiano and Grillner, 2005)]. The contralateral input, when present, must thus be assumed not only to activate the fMN but also to somehow reduce or abolish the latent ipsilateral exci- 


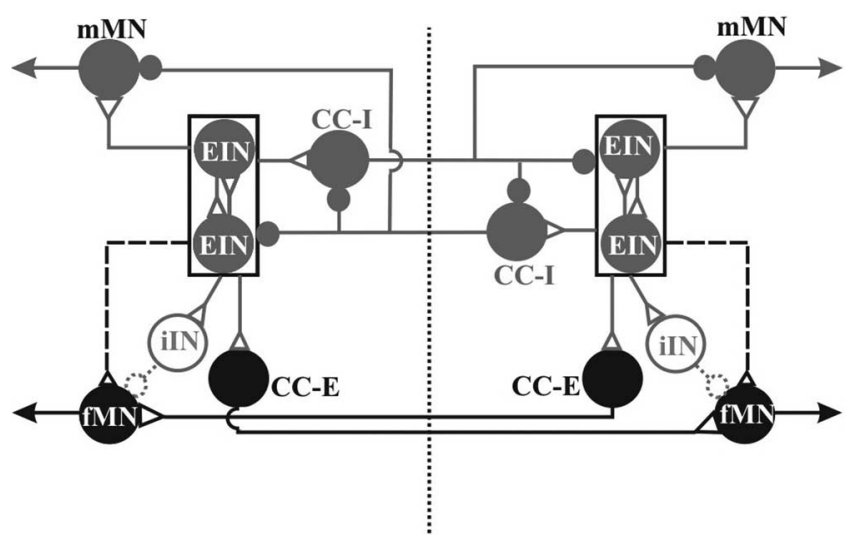

Figure 9. Schematic drawing of pathways possibly contributing to patterning and phasing of fMN activity in one segment of the caudal lamprey spinal cord. For better clarity network topology is presented without distinction between local and intersegmental projections. The excitatory CPG kernel driving mMNs on either side of the spinal cord is depicted as a box. These burst generating kernels are active in antiphase by way of interposed mutually inhibiting interneurons (Cangiano and Grillner, 2003, 2005) [inhibitory contralaterally and caudally projecting interneurons (C(-I)]. The excitatory kernels drive not only ipsilateral mMNs, but also more weakly fMNs. The connection to the fMNs is dashed because this influence is only detected after $50-70 \%$ lesioning of the spinal cord midline as well as in the hemi cord. The specific connection of excitatory CC-E interneurons with fMNs, presented in this study, seems to be monosynaptic. A potential neural source for the in-phasic inhibitory drive that fMNs receive during fictive locomotion is given (stippled ilNs). Gray neurons and/or connections have been described previously; black neurons and/or connections are newly identified; stippled neurons and/or connections are hypothetical.

tatory input to $\mathrm{fMNs}$ and, instead, gate or potentiate ipsilateral inhibition (cf. Figs. $3 A, 8$ ). The change in activity phasing in fMNs revealed after cessation of contralateral signals is reminiscent of findings of Currie and Lee (1996) in the turtle spinal cord. After removal of glycinergic inhibition, an excitatory contralateral component during fictive flexion reflex motor output was unmasked.

Our data therefore show that pathways exist in the lamprey spinal cord, that are able to contribute both to alternation and in-phase activation of ipsilateral $\mathrm{fMN}$ and $\mathrm{mMN}$. Alternation between the two muscle types has been shown in vivo during steady forward swimming in the intact lamprey (Mentel et al., 2006). More complex patterns of coordination, including coactivation have been reported for turning and other maneuvers (Porres et al., 2007), suggesting that both kinds of synaptic drive identified for fMNs in the spinal cord may serve different functional roles during free swimming, under appropriate circumstances.

\section{Common premotor rhythmic networks in the lamprey hemicord for different sets of motoneurons}

The finding that $\mathrm{fMNs}$ and $\mathrm{mMNs}$ in the spinal cord share common ipsilateral premotor networks is not only interesting with respect to understanding of topology and operation of the spinal locomotor network for swimming in the lamprey, but also in its own right. Paired intracellular recordings from $\mathrm{fMNs}$ and $\mathrm{mMNs}$ revealed very similar synaptic inputs during rhythmic activity. Comparing the oscillatory membrane potential trajectories between fMNs and mMNs during electrically released bouts of activity shows that both types of motoneurons receive common, but not identical synaptic inputs. Qualitatively, the gross similarity in membrane potential modulation in both types of motoneurons is equivalent to that observed in segmental mMNs by Wallén et al. (1985) and Buchanan and Kasicki (1999). They found that although the similarities between the membrane potential trajectories of mMNs in the same or adjacent segments could be very prominent, their correlation coefficients varied in a wide range from down to 0.06 up to as high as 0.74 , and had a mean of 0.44 . This is close to the range of values found between $\mathrm{fMN}$ and $\mathrm{mMN}$ in this study. From our results it is clear that in the lamprey spinal cord different sets of motoneurons receive rhythmic synaptic inputs from common premotor networks within the hemicord. How can this finding be placed in the context of the present knowledge on the operation of spinal networks for locomotion?

Cangiano and Grillner $(2003,2005)$ showed that pattern generation for swimming in the lamprey spinal cord results from a hemisegmental glutamatergic network that generates a fast rhythm driving ipsilateral motoneurons. This rhythmicity is then transformed into an alternating activity pattern between both sides of the spinal cord by crossed glycinergic inhibition, which is mediated by inhibitory commissural interneurons (Grillner and Wallén, 1980; Buchanan, 1982, 2001) (Fig. 9). Evidence for a kernel of excitatory neurons generating basic aspects of the burst generation is accumulating for a variety of rhythmic motor networks, not only in vertebrate locomotion, but also breathing or saccadic eye movements (for review, see Kiehn et al., 2006). From these systems the conception emerges that the activity generated by such kernels of excitatory neurons is sculptured to a specific motor pattern on a subsequent level of network interaction. The specific interest here is that we have shown that $\mathrm{AMN}$ can either receive input from such ipsilateral networks and be activated in parallel with mMNs, or they can also be driven by the contralateral burst generating networks via commissural excitatory interneurons with long descending axons.

\section{References}

Alford S, Grillner S (1991) The involvement of $\mathrm{GABA}_{\mathrm{B}}$ receptors and coupled G-proteins in spinal GABAergic presynaptic inhibition. J Neurosci 11:3718-3726.

Brodin L, Grillner S (1985) The role of putative excitatory amino acid neurotransmitters in the initiation of locomotion in the lamprey spinal cord. I. The effects of excitatory amino acid antagonists. Brain Res 360:139-148.

Brodin L, Grillner S, Rovainen CM (1985) N-Methyl-D-Aspartate (NMDA), kainate and quisqualate receptors and the generation of fictive locomotion in the lamprey spinal-cord. Brain Res 325:302-306.

Buchanan JT (1982) Identification of interneurons with contralateral, caudal axons in the lamprey spinal cord: synaptic interactions and morphology. J Neurophysiol 47:961-975.

Buchanan JT (1986) Premotor interneurons in the lamprey spinal cord: morphology, synaptic interaction and activities during fictive swimming. In: Neurobiology of vertebrate locomotion. (Grillner S, Stein PS, Stuart DG, Forssberg H, Herman RM, eds), pp 3212-3333. London: MacMillan. Buchanan JT (2001) Contributions of identifiable neurons and neuron classes to lamprey vertebrate neurobiology. Prog Neurobiol 63:441-466.

Buchanan JT, Cohen A (1982) Activities of identified interneurons, motoneurons, and muscle fibers during fictive swimming in the lamprey and effects of reticulospinal and dorsal cell stimulation. J Neurophysiol 47:948-960.

Buchanan JT, Grillner S (1987) Newly identified "glutamate interneurons" and their role in locomotion in the lamprey spinal cord. Science 236:312-314.

Buchanan JT, Grillner S (1988) A new class of small inhibitory interneurones in the lamprey spinal cord. Brain Res 438:404-407.

Buchanan JT, Kasicki S (1999) Segmental distribution of common synaptic inputs to spinal motoneurons during fictive swimming in the lamprey. J Neurophysiol 82:1156-1163.

Büschges A, Wickström M, Grillner S, El Manira A (2000) Roles of calcium channel subtypes in a vertebrate spinal locomotor network. J Neurophysiol 84:2746-2757.

Cangiano L, Grillner S (2003) Fast and slow locomotor burst generation in the hemispinal cord of the lamprey. J Neurophysiol 89:2931-2942. 
Cangiano L, Grillner S (2005) Mechanisms of rhythm generation in a spinal locomotor network deprived of crossed connections: the lamprey hemicord. J Neurosci 25:923-935.

Cohen AH, Harris-Warrick RM (1984) Strychnine eliminates alternating motor output during fictive locomotion in the lamprey. Brain Res 293:164-167.

Currie SN, Lee S (1996) Glycinergic inhibition in the turtle spinal cord regulates the intensity and pattern of fictive flexion reflex motor output. Neurosci Lett 205:75-78.

El Manira A, Shupliakov O, Fagerstedt P, Grillner S (1996) Monosynaptic input from cutaneous sensory afferents to fin motoneurons in lamprey. J Comp Neurol 369:533-542.

Fagerstedt P, Pavel Z, Deliagina TG, Orlovsky GN, Grillner S (2000) Crossed reciprocal inhibition evoked by electrical stimulation of the lamprey spinal cord. Exp Brain Res 134:147-154.

Grillner S (2003) The motor infrastructure: from ion channels to neuronal networks. Nat Rev Neurosci 4:573-586.

Grillner S, McClellan A, Sigvard K, Wallén P, Wilén M (1981) Activation of NMDA-receptors licits "fictive locomotion" in lamprey spinal cord in vitro. Acta Physiol Scand 113:549-551.

Grillner S, Wallén P (1980) Does the central pattern generation for locomotion in lamprey depend on glycine inhibition? Acta Physiol Scand 110:103-105.

Haugland R (2002) Handbook of fluorescent probes and research products, Ed 9, pp 581-590. Eugene, OR: Molecular Probes.

Kiehn O, Büschges A, Duch C, Grillner S, Isa T, Lansner A. Pflüger, HJ, Richter DW, Sillar KT, Smith J and Sparks DL (2006) Neuromodulation of microcircuits in motor systems of invertebrates. In: Microcircuits: the interface between neurons and global brain function (Grillner S, Graybiel AM, eds), pp 77-103. Cambridge, MA: MIT.

Kremer E, Lev-Tov A (1997) Localization of the spinal network associated with generation of hindlimb locomotion in the neonatal rat and organization of its transverse coupling system. J Neurophysiol 77:1155-1170.

Mentel T, Krause A, Pabst M, El Manira A, Büschges A (2006) Activity of fin muscles and fin motoneurons during swimming motor pattern in the lamprey. Eur J Neurosci 23:2012-2026.

Orlovsky GN, Deliagina TG, Grillner S (1999) Neuronal control of locomotion. Oxford, UK: Oxford UP.

Pérez CT, Hill RH, Grillner S (2007) Endogenous tachykinin release contributes to the locomotor activity in lamprey. J Neurophysiol 97:3331-3339.

Porres C, Pabst M, Büschges A, Mentel T (2007) Recruitment and activity of fin muscles during swimming maneuvers in lamprey. 100th Annual Meeting of the Deutsche Zoologische Gesellschaft, Cologne, Germany, September.

Rovainen CM, Birnberger KL (1971) Identification and properties of motoneurons to fin muscle of the sea lamprey. J Neurophysiol 34:974-982.

Shupliakov O, Wallén P, Grillner S (1992) Two types of motoneurons supplying dorsal fin muscles in lamprey and their activity during fictive locomotion. J Comp Neurol 321:112-123.

Wallén P, Williams TL (1984) Fictive locomotion in the lamprey spinal cord in vitro compared with swimming in the intact and spinal animal. J Physiol (Lond) 347:225-239.

Wallén P, Grillner S, Feldman JL, Bergelt S (1985) Dorsal and ventral myotome motoneurons and their input during fictive locomotion in lamprey. J Neurosci 5:654-661. 